\title{
FINITE ELEMENT APPROXIMATION OF AEROELASTIC PROBLEMS WITH CONSIDERATION OF NONLINEAR EFFECTS DUE TO LARGE DISPLACEMENTS
}

\author{
Petr Sváček ${ }^{1}$ \\ ${ }^{1}$ Department of Technical Mathematics, Faculty of Mechanical Engineering, Czech Technical \\ University in Prague, Technická 4, 16000 Praha 6, Czech Republic
}

\begin{abstract}
This paper focus on numerical approximation of aeroelastic problems. In particular the twodimensional incompressible viscous fluid flow interaction with an elestic structure is considered. The structure motion is described with the aid of nonlinear ordinary differential equations describing either the motion of a flexibly supported airfoil or the motion of a flexibly supported airfoil with a control section. The aerodynamic forces are modelled either by the low-fidelity linearized approach or by high fidelity models based on approximation of these forces based on the evaluated flow quantities. The flow is modelled by the system of Navier-Stokes equations. The time-dependent computational domain is taken into account by the Arbitrary LagrangianEulerian method, where the stabilized finite element method is applied. Numerical results are presented.
\end{abstract}

Keywords: nonlinear aeroelasticity, finite element method

\section{Introduction}

The aeroelastic problems or fluid-structure interactions play an important role in many technical applications. Let us mention aeroelastic problems in aerospace engineering, see e.g. [3], or aeroelastic stability of bridges and tall buildings in civil engineering, see e.g. [13]. Aeroelasticians in practice use the linearized methods to determine the aeroelastic stability region, see [9]. The use of linearized aerodynamics allows to determine the aeroelastic asymptotic stability in terms, see [3], [4], or [2]. These approaches are well studied and the asymptotic stability approach is widely used as it allows to determine the critical velocity. On the other hand the aeroelastic stability does not always guarantee safety as e.g. an external excitation can lead to transient growth and consequently to the structural failure, see $[16,6]$. In such cases use of high-fidelity models can be attractive as it can provide more detailed information. However, the mathematical modelling of FSI problems in general is much more complicated, because one needs to consider the viscous possibly turbulent flow which is moreover in the interaction with the nonlinear behavior of the elastic structure. Moreover, the time changes of the flow domain due to the vibrating structure cannot be neglected and the methods applicable on moving domain or meshes must be employed, see e.g. [7]. Last, the coupled fluid-structure system is usually solved with the aid of the so-called partitioned or de-coupled approach, which approximates the fluid flow and structure motion by different solvers. This allows to use optimized solvers, but on the other hand such a coupling can possibly lead to an artificial instability of such a scheme, see e.g. [5]. In order to overcome this phenomena either the partitioned approach with relaxed strongly coupled algorithm or the monolithic approaches can be used, cf. [1, 8] or [10].

This paper focus on overview of results obtained by finite element method and its comparison with the linearized approaches. The numerical method based on a fully stabilized finite element method is used using the combination of the streamline-upwind/Petrov-Galerkin(SUPG), the pressure-stabilizing/Petrov-Galerkin (PSPG) methods, and the grad-div stabilization, see e.g. [12]. The arbitrary Lagrangian-Eulerian method is used to treat the motion of the computational domain, see [11]. The nonlinear behaviour of the structure is considered for the post-critical regime. 


\section{Equations of Motion}

Equations of motion of an elastic structure immersed in fluid are in many cases written with the aid of independent variables $\boldsymbol{q}(t)=\left(q_{1}, \ldots, q_{n}\right)^{T}$ as

$$
\mathbb{M} \ddot{\boldsymbol{q}}+\mathbb{D} \dot{\boldsymbol{q}}+\mathbb{K} \boldsymbol{q}=\boldsymbol{F}
$$

where $\mathbb{M}$ denotes the mass matrix, $\mathbb{K}$ is the stiffness matrix and $\mathbb{D}$ is the structural damping matrix written using the proportional damping approach as

$$
\mathbb{D}=\xi_{2} \mathbb{K}+\xi_{1} \mathbb{M}
$$

where $\xi_{1}, \xi_{2} \geq 0$ are suitably chosen parameters. Furhter, in Eq. (1) by $\boldsymbol{F}$ the aerodynamical forces acting on the system are denoted.

In general the matrices $\mathbb{M}, \mathbb{K}$ and $\mathbb{D}$ can depend on the vector $\boldsymbol{q}$, i.e. $\mathbb{M}=\mathbb{M}(\boldsymbol{q}), \mathbb{K}=$ $\mathbb{K}(\boldsymbol{q})$ and $\mathbb{D}=\mathbb{D}(\boldsymbol{q})$. In this case the system of equations (1) is possibly nonlinear and for the purpose of asymptotic stability the linearized system of equations is considered using matrices $\mathbb{M}=\mathbb{M}\left(\boldsymbol{q}_{0}\right), \mathbb{K}=\mathbb{K}\left(\boldsymbol{q}_{0}\right)$ and $\mathbb{D}=\mathbb{D}\left(\boldsymbol{q}_{0}\right)$ for a suitable choice $\boldsymbol{q}_{0}$.
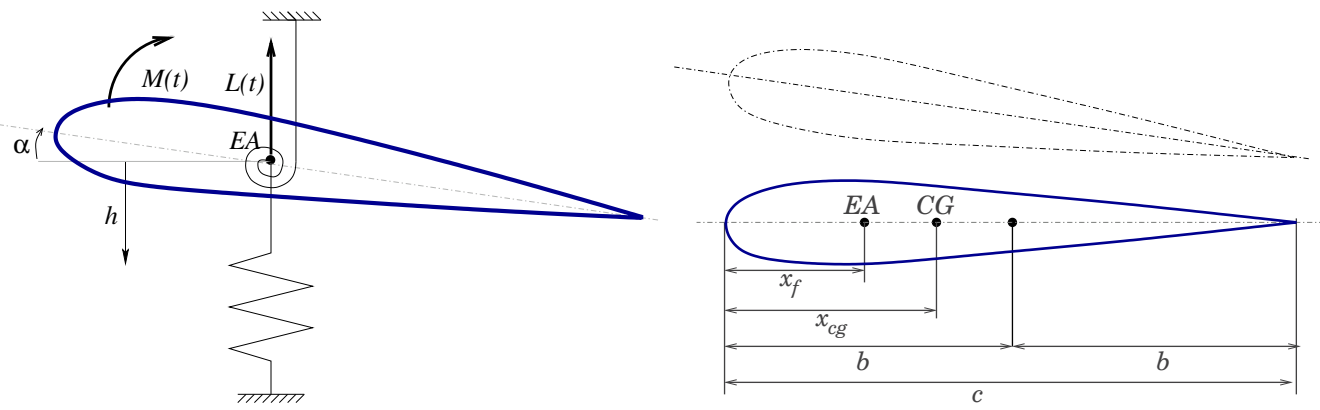

Figure 1: Elastically supported airfoil with two degrees of freedom bending $h$ and pitching $\alpha$ (on the left). Airfoil in undeformed position showing the elastic axis (EA) and center of gravity (CG) (on the right).

\subsection{Equations of Motion for Airfoil with Two Degrees of Freedom}

First, an airfoil section with the chord $c=2 b$ is considered, which can be vertically displaced by $h$ (downwards positive) and rotated by angle $\alpha$ (clockwise positive), see Fig. 1 . The elastic axis in the undeformed position is located at $x_{f}$ from the leading edge and the center of gravity is located at $x_{c g}$ from the leading edge. These two degrees of freedom $h$ and $\alpha$ are also called the bending and the pitching, respectively. The nonlinear equations of motion are then given by (see [14])

$$
\begin{array}{r}
m \ddot{h}+S_{\alpha} \ddot{\alpha} \cos \alpha-S_{\alpha} \dot{\alpha}^{2} \sin \alpha+k_{h} h=-L(t), \\
S_{\alpha} \ddot{h} \cos \alpha+I_{\alpha} \ddot{\alpha}+k_{\alpha} \alpha=M(t) .
\end{array}
$$

where $m$ is the mass of the airfoil, $S_{\alpha}$ is the static moment around the elastic axis (EA), and $I_{\alpha}$ is the inertia moment around EA. The parameters $k_{h}$ and $k_{\alpha}$ denote the stiffness coefficients. On the right-hand side the aerodynamical lift force $L(t)$ and aerodynamical torsional moment $M(t)$ are involved.

Under the assumption of small displacements (i.e. $\alpha \approx 0, \sin \alpha \approx 0$ and $\cos \alpha \approx 1$ ) the system of equations can be linearized as system (1) for $\boldsymbol{q}=(h, \alpha)^{T}$, where

$$
\mathbb{M}=\left(\begin{array}{rr}
m & S_{\alpha} \\
S_{\alpha} & I_{\alpha}
\end{array}\right), \quad \mathbb{K}=\left(\begin{array}{rr}
k_{h} & 0 \\
0 & k_{\alpha}
\end{array}\right), \quad \boldsymbol{F}=\left(\begin{array}{c}
-L(t) \\
M(T)
\end{array}\right) .
$$

In order to solve system of ordinary differential equations $(3)$ or $(1,4)$ the aerodynamical lift force $L(t)$ and the aerodynamical moment $M(t)$ needs to be evaluated for any time instant $t$. These aerodynamical forces can be expressed as

$$
L=-l \int_{\Gamma_{W t}} \sigma_{2 j} n_{j} d S, \quad M=l \int_{\Gamma_{W t}} \sigma_{i j} n_{j} r_{i}^{\text {ort }} d S,
$$


where $l$ is the depth of the airfoil section, $\sigma_{i j}$ are the components of the fluid stress tensor given as $\sigma_{i j}=\left[-p \delta_{i j}+\mu\left(\frac{\partial u_{i}}{\partial x_{j}}+\frac{\partial u_{j}}{\partial x_{i}}\right)\right]$, where $p$ is the pressure, $\mu$ is the (dynamic) viscosity, $\boldsymbol{u}=\left(u_{1}, u_{2}\right)^{T}$ is the flow velocity, $x^{\mathrm{EA}}=\left(x_{1}^{\mathrm{EA}}, x_{2}^{\mathrm{EA}}\right)$ is the position of EA of the airfoil at the time instant $t$ and $r_{1}^{\text {ort }}=-\left(x_{2}-x_{2}^{\mathrm{EA}}\right), r_{2}^{\text {ort }}=x_{1}-x_{1}^{\mathrm{EA}}$. The system of equations is completed by the prescription of suitable initial conditions at $t_{0}=0$ for $\alpha\left(t_{0}\right), h\left(t_{0}\right), \dot{\alpha}\left(t_{0}\right)$ and $\dot{h}\left(t_{0}\right)$.

\subsection{Airfoil with three degrees of freedom}

Second, a typical airfoil with semichord $b$ immersed in subsonic air flow is considered as shown in Figure 2. A trailing edge flap is hinged at $c_{\beta} b$ after of the midchord. By $h, \alpha$ and $\beta$ the plunging of the elastic axis, pitching of the airfoil and rotation of the flap is denoted, see Figure 2. The system motion generates unsteady aerodynamic lift $L=L(t)$, aerodynamical moment $M=M(t)$ and hinge moment $M_{\beta}=M_{\beta}(t)$. By $k_{h}, k_{\alpha}$ and $k_{\beta}$ we denote the spring constant of wing bending, wing torsional stiffness and flap hinge moment. The mass part of the model is defined by the mass $m$ and the moment of inertia $I_{\alpha}$ of the total airfoil about the elastic axis. The flap moment of inertia about the hinge is defined as $I_{\beta}$. The linear equations of motion for the coordinates $\boldsymbol{q}=(h, \alpha, \beta)$ are given by $(1)$ where

$$
\mathbb{M}=\left(\begin{array}{ccc}
m & S_{\alpha} & S_{\beta} \\
S_{\alpha} & I_{\alpha} & S_{\beta}\left(x_{c s}-x_{f}\right)+I_{\alpha} \\
S_{\beta} & S_{\beta}\left(x_{c s}-x_{f}\right)+I_{\alpha} & I_{\beta}
\end{array}\right), \quad \mathbb{K}=\left(\begin{array}{ccc}
k_{h} & 0 & 0 \\
0 & k_{\alpha} & 0 \\
0 & 0 & k_{\beta}
\end{array}\right),
$$

and $\boldsymbol{F}=\left(L, M, M_{\beta}\right)^{T}$, where the aerodynamical lift force $L$ and the aerodynamical moment $M$ are expressed by Eq. (4) with $\Gamma_{W t}=\Gamma_{B t} \cup \Gamma_{F t}$ and the aerodynamical moment of the control section $\Gamma_{F t}$ is given by

$$
M_{\beta}=l \int_{\Gamma_{F t}} \sigma_{i j} n_{j} r_{i}^{\mathrm{F}, \mathrm{ort}} d S,
$$

where $r_{1}^{\mathrm{F}, \mathrm{ort}}=-\left(x_{2}-x_{2}^{\mathrm{EF}}\right), r_{2}^{\mathrm{F}, \text { ort }}=x_{1}-x_{1}^{\mathrm{EF}}$.
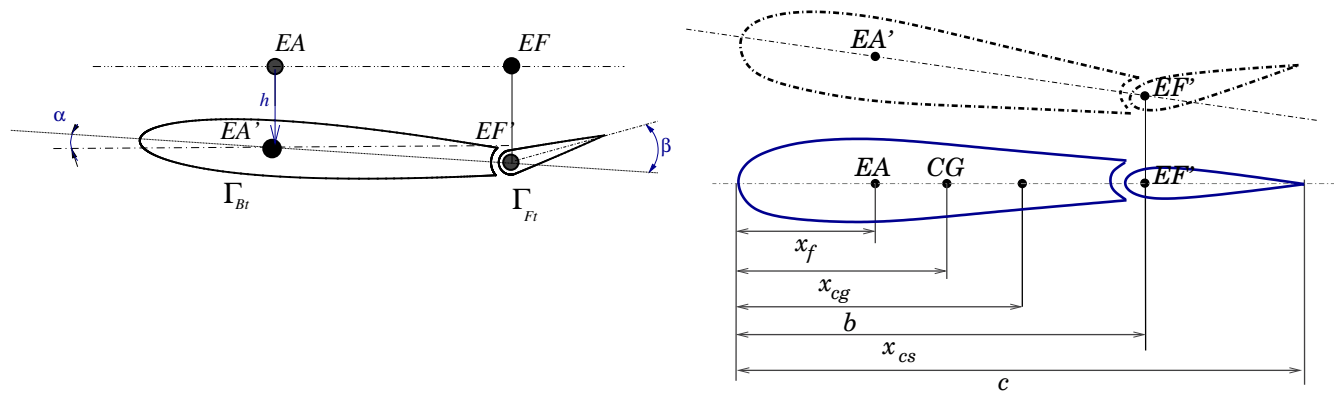

Figure 2: Typical airfoil section for the case of three degrees of freedom: Here, $c$ denotes the chord of the airfoil with $b=c / 2$ being the semichord, $e b$ denotes the location of the elastic axis after the midchord and $x_{\alpha} b$ is the location of the center of gravity after the elastic axis is denoted. By $c_{\beta} b$ the location of the flap hinge after the midchord is denoted and by $x_{\beta} b$ the location of the center of gravity of the flap is denoted.

\section{Aerodynamic forces evaluation}

For the sake of brevity the evaluation of aerodynamical forces is presented here only for the first case of airfoil with 2DOF. The low-fidelity approach is based on the linearized aerodynamics, see [15]. The high fidelity approach is based on the finite element approximations of incompressible viscous fluid flow, see [14]. In this case the aerodynamical lift force $L(t)$ and aerodynamical torsional moment $M(t)$ can be evaluated using flow quantities by Eqs. (4) In order to evaluate the forces by (4), flow velocity and pressure needs to be evaluated using the flow model. 


\subsection{Low-fidelity model}

Let us denote by $c$ and by $b$ the chord and the midchord $b=c / 2$ of the airfoil, respectively. Further, by ce let us denote the distance of the elastic axis from the aerodynamical center, i.e. $c e=x_{f}-\frac{c}{4}$. Using the assumption of harmonic motion of the airfoil, with the angular frequency $\omega$, the aerodynamical forces are given as sum of circulatory and non-circulatory parts, see e.g. [2]), i.e

$$
\begin{aligned}
\frac{L}{\pi \rho b} & =b\left(\ddot{h}-\left(x_{f}-b\right) \ddot{\alpha}+U_{\infty} \dot{\alpha}\right)+2 U_{\infty} C(k)\left(\dot{h}+U_{\infty} \alpha+\left(\frac{3}{2} b-x_{f}\right) \dot{\alpha}\right) \\
\frac{M}{\pi \rho b^{2}} & =\left(\left(x_{f}-b\right) \ddot{h}-\left(\frac{b^{2}}{8}+\left(x_{f}-b\right)^{2}\right) \ddot{\alpha}-U_{\infty}\left(\frac{3}{2} b-x_{f}\right) \dot{\alpha}\right) \\
& +\frac{2 U_{\infty}}{b}\left(x_{f}-\frac{b}{2}\right) C(k)\left(\dot{h}+U_{\infty} \alpha+\left(\frac{3}{2} b-x_{f}\right) \dot{\alpha}\right),
\end{aligned}
$$

where $x_{f}$ denotes the distance of the elastic axis from the leading edge, $b=\frac{c}{2}$ denotes the midchord of the airfoil, $\rho$ denotes the constant density of the fluid, $U_{\infty}$ denotes the far field velocity, $C(k)$ is the Theodorsen function and $k$ is the reduced frequency $k=b \omega / U_{\infty}$. Using (7) in the system of equations (3) leads to the system of two nonlinear equations with two parameters, the reduced frequency $k$ and the far field velocity $U_{\infty}$. Based on this still nonlinear system - $(7)$ is valid only for the case of harmonic motion - the critical flutter velocity can be determined and using the so-called $p-k$ method the vibration frequency and damping for a given flow velocity $U_{\infty}$ can be computed, see e.g. [4].

\section{Incompressible viscous fluid flow model}

The flow in the bounded time-dependent domain $\Omega_{t} \subset R^{2}$ is governed by the incompressible Navier-Stokes equations written in the ALE form

$$
\rho \frac{D^{\mathcal{A}} \boldsymbol{u}}{D t}+\rho\left(\left(\boldsymbol{u}-\boldsymbol{w}_{D}\right) \cdot \nabla\right) \boldsymbol{u}+\nabla p-\nabla \cdot\left(\mu\left(\nabla \boldsymbol{u}+(\nabla \boldsymbol{u})^{T}\right)\right)=0, \quad \nabla \cdot \boldsymbol{u}=0,
$$

where $\boldsymbol{u}=\boldsymbol{u}(x, t)$ is the flow velocity, $p=p(x, t)$ is the pressure, $\rho$ is the constant fluid density, $\mu$ is the fluid viscosity, $\frac{D^{\mathcal{A}}}{D t}$ denotes the ALE derivative and $\boldsymbol{w}_{D}$ denotes the domain velocity, see e.g. [14]. The boundary of the computational domain $\partial \Omega_{t}$ consists of mutually disjoint parts $\Gamma_{I}$ (inlet), $\Gamma_{O}$ (outlet), $\Gamma_{W t}$ (the moving part, vibrating airfoil). The following boundary conditions are prescribed
a) $\boldsymbol{u}=\boldsymbol{w}_{D}$ on $\Gamma_{W t}$,
b) $\boldsymbol{u}=\mathbf{v}_{D}$ on $\Gamma_{I}$,
c) $-p \boldsymbol{n}+\frac{1}{2} \rho(\boldsymbol{u} \cdot \boldsymbol{n})^{-} \boldsymbol{u}+\mu \frac{\partial \boldsymbol{u}}{\partial \boldsymbol{n}}=0$, on $\Gamma_{O}$,

where $\boldsymbol{n}$ denotes the unit outward normal vector to $\Omega_{t}$ and $\alpha^{-}=\min (\alpha, 0)$ denotes the negative part of a real number $\alpha$. The computations start from the initial condition $\boldsymbol{u}(x, 0)=\boldsymbol{u}^{0}(x)$ for $x \in \Omega_{0}$.

\section{Numerical approximation}

In order to discretize the problem (8) in time, the equidistant partition $0=t_{0}<t_{1}<\cdots<$ $T, t_{k}=k \Delta t$ of the time interval $[0, T]$ is considered with the constant time step $\Delta t>0$. The time derivative in (8) is approximated by the second order backward difference formula

$$
\left.\frac{D^{\mathcal{A}} \boldsymbol{u}}{D t}\right|_{t=t_{n+1}} \approx \frac{3 \boldsymbol{u}^{n+1}-4 \tilde{\boldsymbol{u}}^{n}+\tilde{\boldsymbol{u}}^{n-1}}{2 \Delta t} .
$$

The time discretized problem is than spatially discretized by the finite element method. To this end the approximations $\boldsymbol{u}^{k}$ and $p^{k}$ are sought in the finite element spaces $\boldsymbol{W}_{h}$ and $Q_{h}$, respectively. By symbol $\boldsymbol{X}_{h}$ the space of test functions is denoted $\boldsymbol{X}_{h} \subset \boldsymbol{W}_{h}$. In the practical computations these 
spaces are defined with the aid an admissible triangulation $\mathcal{T}_{h}$ of $\Omega$ and the well known Taylor-Hood finite element is used. This means that the fluid velocity is approximated by continuous piecewise quadratic functions and the pressure is approximated by continuous piecewise linear functions.

The discrete formulation contains the Galerkin terms defined for any $U=(\boldsymbol{u}, p)$ and $V=(\boldsymbol{\varphi}, q)$ by

$$
\begin{aligned}
a(U, V) & =\left(\rho \frac{3 \boldsymbol{u}}{2 \Delta t}+\rho\left(\left(\boldsymbol{u}-\boldsymbol{w}_{D}\right) \cdot \nabla\right) \boldsymbol{u}, \boldsymbol{\varphi}\right)_{\Omega}+(\mu \nabla \boldsymbol{u}, \nabla \boldsymbol{\varphi})_{\Omega}-(p, \nabla \cdot \boldsymbol{\varphi})_{\Omega}+(\nabla \cdot \boldsymbol{u}, q)_{\Omega},(11) \\
f(V) & =\left(\rho \frac{4 \tilde{\boldsymbol{u}}^{n}-\tilde{\boldsymbol{u}}^{n-1}}{2 \Delta t}, \boldsymbol{\varphi}\right)_{\Omega} .
\end{aligned}
$$

The stabilization terms containing the SUPG/PSPG stabilization are defined by

$$
\begin{aligned}
\mathcal{L}(U, V) & =\sum_{K \in \mathcal{T}_{h}} \delta_{K}\left(\rho \frac{3 \boldsymbol{u}}{2 \Delta t}-\mu \Delta \boldsymbol{u}+\rho\left(\left(\boldsymbol{u}-\boldsymbol{w}_{D}\right) \cdot \nabla\right) \boldsymbol{u}+\nabla p, \rho\left(\left(\boldsymbol{u}-\boldsymbol{w}_{D}\right) \cdot \nabla\right) \boldsymbol{\varphi}+\nabla q\right)_{K} \\
\mathcal{F}(V) & =\sum_{K \in \mathcal{T}_{h}} \delta_{K}\left(\rho \frac{4 \tilde{\boldsymbol{u}}^{n}-\tilde{\boldsymbol{u}}^{n-1}}{2 \Delta t},(\boldsymbol{u} \cdot \nabla) \boldsymbol{\varphi}+\nabla q\right)_{K},
\end{aligned}
$$

and the stabilization term for div-div stabilization reads $\mathcal{P}(U, V)=\sum_{K \in \mathcal{T}_{h}} \tau_{K}(\nabla \cdot \boldsymbol{u}, \nabla \cdot \varphi)_{K}$ with the choice of $\delta_{K}$ and $\tau_{K}$ according to [14]. This approach was previously numerically verified on a number of benchmark problems, see [14], and also it satisfy the optimal error estimates and thus it should be more precise compared e.g. to the classical finite volume methods.

Then the non-linear stabilized discrete problem at a time instant $t=t^{n+1}$ reads: Find $p^{n+1}:=p$, $\boldsymbol{u}^{n+1}:=\boldsymbol{u}, U=(\boldsymbol{u}, p) \in \boldsymbol{W}_{h} \times Q_{h}$, such that $\boldsymbol{u}$ satisfies approximately the conditions (9 a-b) and

$$
a(U, V)+\mathcal{L}(U, V)+\mathcal{P}(U, V)=f(V)+\mathcal{F}(V),
$$

holds for all $V=(\boldsymbol{\varphi}, q) \in \boldsymbol{X}_{h} \times Q_{h}$. The solution of the nonlinear problem is realized by the Oseen linearization. The nonlinear problem (14) is then coupled with the system (3) and solved simultaneously.

\section{$6 \quad$ Numerical results}

For the numerical simulation the airfoil NACA 0012 was considered in interaction with incompressible fluid with the constant density $\rho=1.225 \mathrm{~kg} / \mathrm{m}^{3}$, the fluid kinematic viscosity $\nu=\mu / \rho=$ $1.5 \times 10^{-5} \mathrm{~m}^{2} \mathrm{~s}$, the airfoil chord $c=0.254 \mathrm{~m}$, the airfoil airfoil mass $m=4.7174 \mathrm{~kg}$, the airfoil inertia moment $I_{a}=0.029522 \mathrm{~kg} \mathrm{~m}{ }^{2}$, the airfoil static moment $S_{a}=0.14978 \mathrm{~kg} \mathrm{~m}$, the bending stiffness was $k_{h}=14741 \mathrm{~N} / \mathrm{m}$ and the torsional stiffness $k_{a}=121.30 \mathrm{~N} \mathrm{~m} / \mathrm{rad}$. The elastic axis was located at 42.5 percent of the airfoil chord measured from the leading edge of the airfoil, $x_{f}=0.425 c=0.10795 \mathrm{~m}$ and similarly the center of gravity of the airfoil was located at 55 percent of the airfoil, i.e. $x_{c g}=0.55 c=0.13970 \mathrm{~m}$.

The aeroelastic system was investigated using the flutter determinant and the flutter velocity was determined as $U_{\text {crit }}=27.387 \mathrm{~m} / \mathrm{s}$ with the flutter frequency $f=9.3181 \mathrm{~Hz}$. The application of the $p-k$ method lead to the determined flutter velocity $U_{c r i t}=27.8 \mathrm{~m} / \mathrm{s}$ with the found flutter frequency $f=9.4 \mathrm{~Hz}$, which agrees well with the flutter determinant method. Let us also mention that for this case the divergence critical velocity is much higher $\left(U_{\text {div }}=52.8 \mathrm{~m} / \mathrm{s}\right)$ then the flutter velocity as the position of the elastic is before the centre of gravity.

The finite element method was applied for solution of this aeroelastic system for the far field velocities in the range $U_{\infty}=5-28 \mathrm{~m} / \mathrm{s}$. Results in terms of aeroelastic response $h$ and $\alpha$ in dependence on $t$ are shown in Figures 3-8. The aeroelastic response agrees well with the linear theory for lower flow velocities $0-20 \mathrm{~m} / \mathrm{s}$ in frequency, see Fig. 9. Also the increase of the aerodynamical damping can be clearly observed.

\section{Conclusion}

In this paper two possible strategies of solution of an classical aeroelastic problem was presented, applied to an aeroelastic problem and the results were discussed. The numerical approximation of 

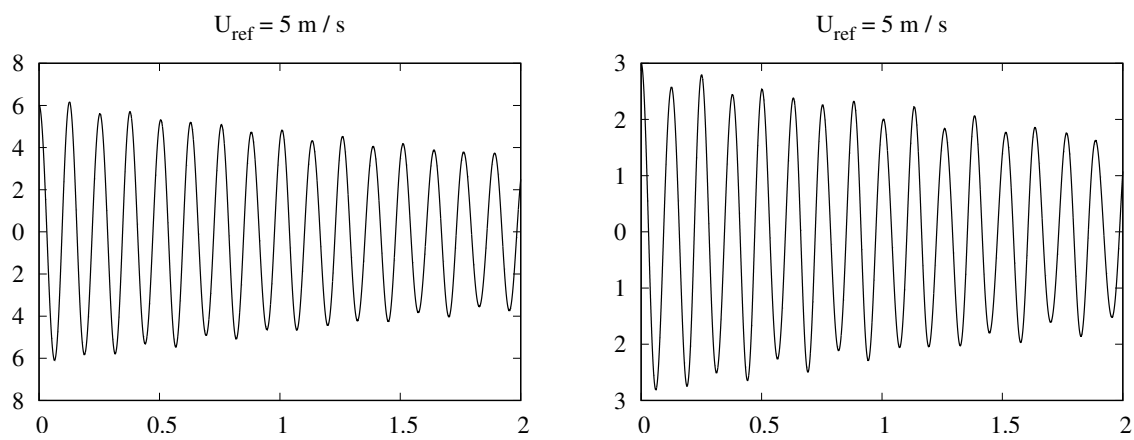

Figure 3: Aeroelastic response for the seconde considered case in terms of $h$ and $\alpha$ in time domain for far field velocity $U_{\infty}=5 \mathrm{~m} / \mathrm{s}$
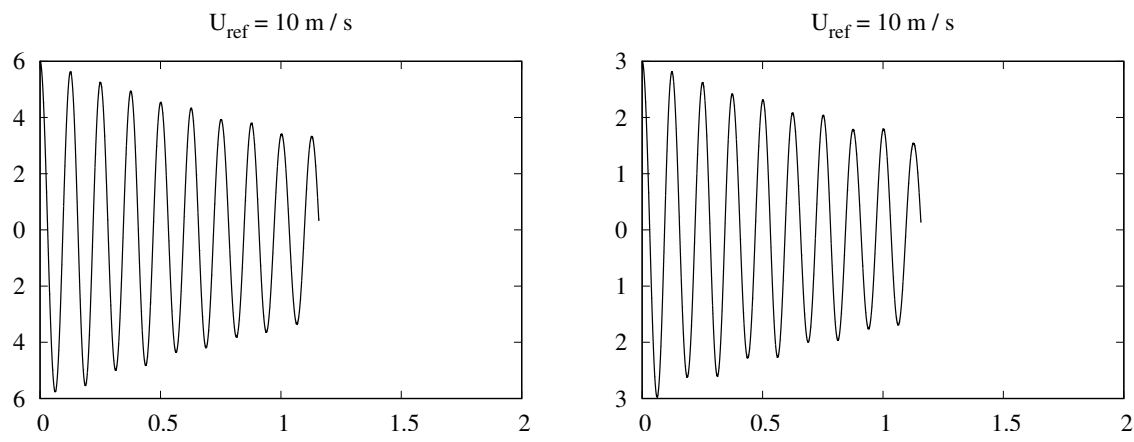

Figure 4: Aeroelastic response for the seconde considered case in terms of $h$ and $\alpha$ in time domain for far field velocity $U_{\infty}=10 \mathrm{~m} / \mathrm{s}$
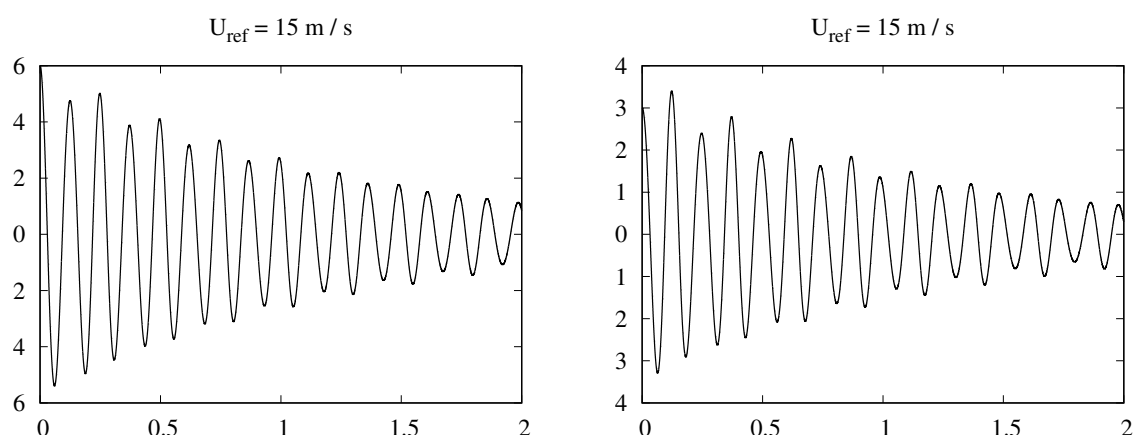

Figure 5: Aeroelastic response for the seconde considered case in terms of $h$ and $\alpha$ in time domain for far field velocity $U_{\infty}=15 \mathrm{~m} / \mathrm{s}$
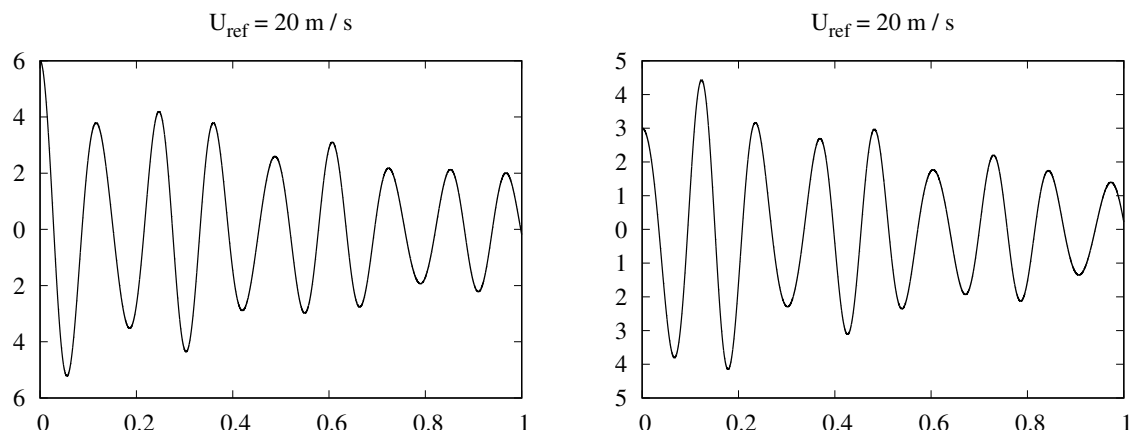

Figure 6: Aeroelastic response for the seconde considered case in terms of $h$ and $\alpha$ in time domain for far field velocity $U_{\infty}=20 \mathrm{~m} / \mathrm{s}$ 

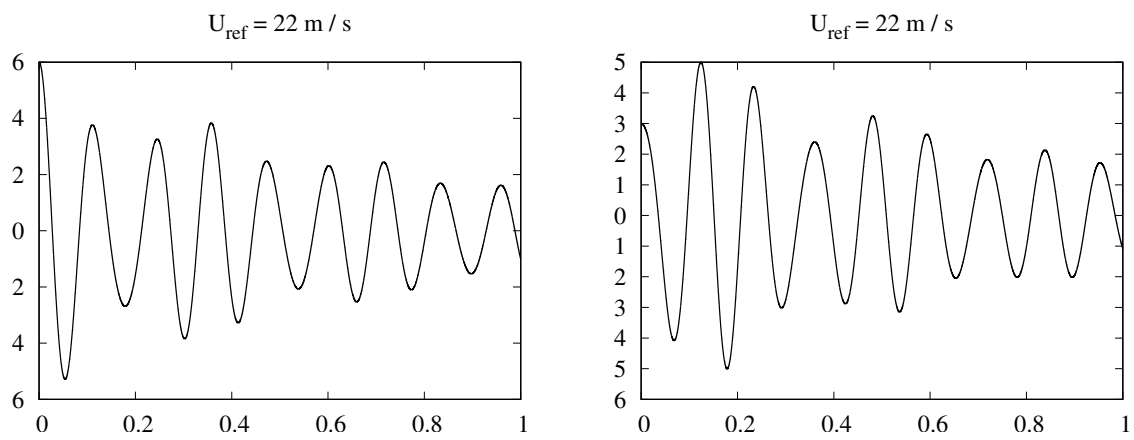

Figure 7: Aeroelastic response for the seconde considered case in terms of $h$ and $\alpha$ in time domain for far field velocity $U_{\infty}=22 \mathrm{~m} / \mathrm{s}$
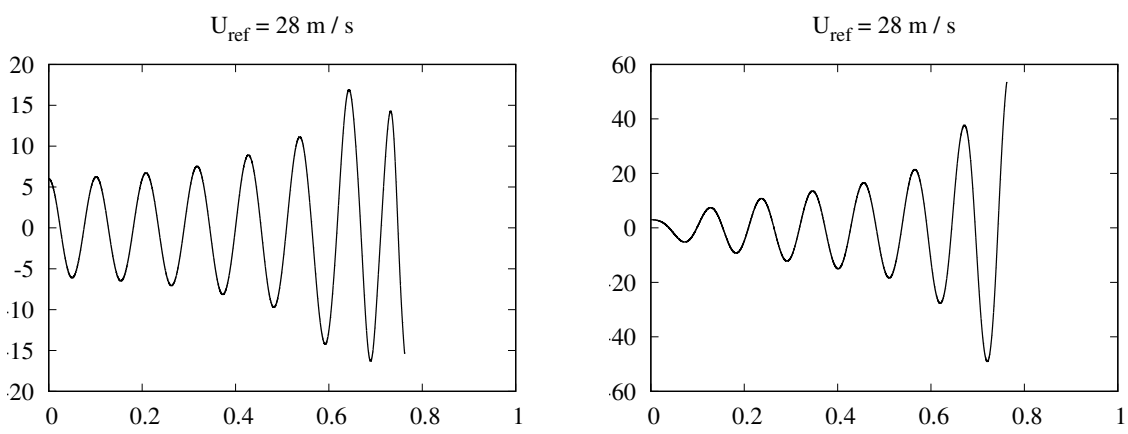

Figure 8: Aeroelastic response for the seconde considered case in terms of $h$ and $\alpha$ in time domain for far field velocity $U_{\infty}=28 \mathrm{~m} / \mathrm{s}$
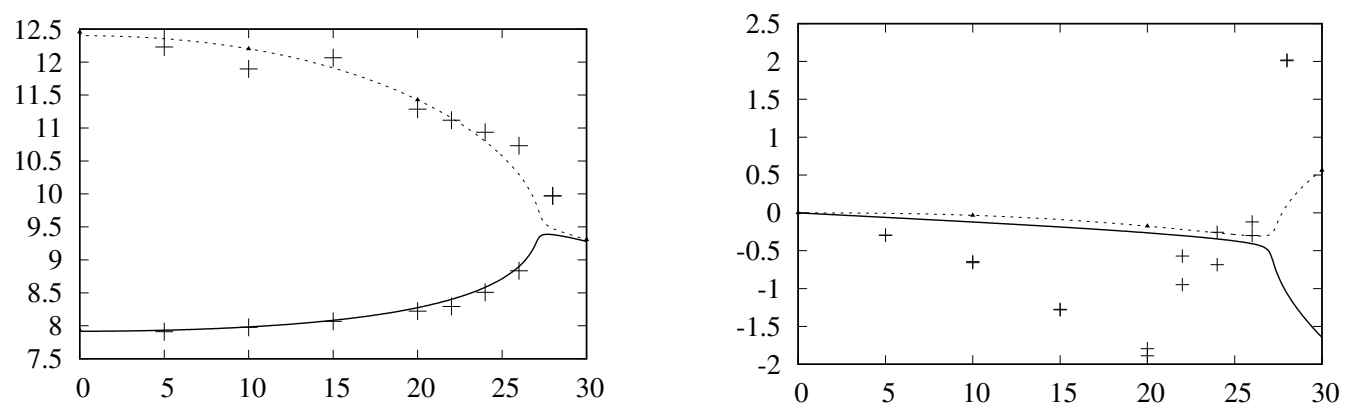

Figure 9: The frequency of vibrating airfoil (on the left) and the aerodynamical damping (on the right) in dependence on the far field velocity $U_{\infty}$. The results obtained by the linearized aerodynamical theory (solid line) and by the finite element method (symbols) are shown.

the nonlinear equations was shown to have the ability to approximate well the sub-critical behaviour of the aeroelastic system. Moreover, by solution of this system also the non-linear behaviour for post-critical far field velocity is possible.

\section{Acknowledgment}

The financial support for the present project was provided by the Centre of Advanced Aircraft Technologies Project No. CZ.02.1.01/0.0/0.0/16_019/0000826. 


\section{References}

[1] E. Aulisa, G. Bornia, and S. Manservisi: Boundary control problems in convective heat transfer with lifting function approach and multigrid Vanka-type solvers. Communications in Computational Physics, 18(3):621-649, 2015.

[2] R.L. Bisplinghoff, H. Ashley, and R.L. Halfman: Aeroelasticity. Dover Books on Aeronautical Engineering Series. Dover Publications, 1996.

[3] Earl H. Dowell and Robert N. Clark: A modern course in aeroelasticity. Solid mechanics and its applications. Kluwer Academic Publishers, Dordrecht, Boston, 2004.

[4] Y. Fung: An Introduction to the Theory of Aeroelasticity. Dover Phoenix Edition: Engineering Dover Publications, 2002.

[5] Christiane Förster, Wolfgang A. Wall, and Ekkehard Ramm: Artificial added mass instabilities in sequential staggered coupling of nonlinear structures and incompressible viscous flows. Computer Methods in Applied Mechanics and Engineering, 196:1278-1293, 2007.

[6] D. Jones and A. Gaitonde: Future fast methods for loads calculations: The 'FFAST' project. In D. Knorzer and J. Szodruch, editors, Innovation for Sustainable Aviation in a Global Environment, pages 110-115. IOS Press BV, 2012.

[7] P. Le Tallec and J. Mouro: Fluid structure interaction with large structural displacements. Computer Methods in Applied Mechanics and Engineering, 190:3039-3067, 2001.

[8] S. Manservisi: Numerical analysis of vanka-type solvers for steady Stokes and Navier-Stokes flows. SIAM Journal on Numerical Analysis, 44(5):2025-2056, 2006.

[9] E. Naudasher and D. Rockwell: Flow-Induced Vibrations. A.A. Balkema, Rotterdam, 1994.

[10] F. Nobile and C. Vergara: An effective fluid-structure interaction formulation for vascular dynamics by generalized robin conditions. SIAM Journal on Scientific Computing, 30:731-763, 2008.

[11] T. Nomura and T. J. R. Hughes: An arbitrary Lagrangian-Eulerian finite element method for interaction of fluid and a rigid body. Computer Methods in Applied Mechanics and Engineering, 95:115-138, 1992.

[12] M. Olshanskii, G. Lube, T. Heister, and J. Löwe: Grad-div stabilization and subgrid pressure models for the incompressible navier-stokes equations. Computer Methods in Applied Mechanics and Engineering, 198(49-52):3975-3988, 2009.

[13] Emil Simiu and Robert H Scanlan: Wind effects on structures : fundamentals and applications to design. New York : John Wiley, 3rd ed edition, 1996.

[14] P. Sváček, M. Feistauer, and J. Horáček: Numerical simulation of flow induced airfoil vibrations with large amplitudes. Journal of Fluids and Structures, 23(3):391-411, 2007.

[15] T. Theodorsen and I.E. Garrick: Flutter calculations in three degrees of freedom. Technical report, NACA Report 1942-741, 1942.

[16] C.V. Verhoosel, T.P. Scholcz, S.J. Hulshoff, and M.A. Gutierrez: Transient stability analysis of an aeroelastic problem with random fluid and structure properties. In Proc of the 10th International Conference on Structural Safety and Reliability, ICOSSAR10, pages 377-384, Osaka, Japan, 2009. 